Kansas State University Libraries

New Prairie Press

\title{
A COMPARISON OF ANALYTIC AND BAYESIAN APPROACHES FOR CHARACTERIZING THERMAL HYSTERESIS IN CATTLE USING ALGEBRAIC AND GEOMETRIC DISTANCES
}

F. Yang

A. M. Parkhurst

S. Zhang

C. N. Lee

T. M. Brown-Brandl

See next page for additional authors

Follow this and additional works at: https://newprairiepress.org/agstatconference

Part of the Agriculture Commons, and the Applied Statistics Commons (c) (1) $9 \odot$

This work is licensed under a Creative Commons Attribution-Noncommercial-No Derivative Works 4.0 License.

\section{Recommended Citation}

Yang, F.; Parkhurst, A. M.; Zhang, S.; Lee, C. N.; Brown-Brandl, T. M.; Gebremedhin, K. G.; and Hillman, P. E. (2012). "A COMPARISON OF ANALYTIC AND BAYESIAN APPROACHES FOR CHARACTERIZING THERMAL HYSTERESIS IN CATTLE USING ALGEBRAIC AND GEOMETRIC DISTANCES," Conference on Applied Statistics in Agriculture. https://doi.org/10.4148/2475-7772.1028

This is brought to you for free and open access by the Conferences at New Prairie Press. It has been accepted for inclusion in Conference on Applied Statistics in Agriculture by an authorized administrator of New Prairie Press. For more information, please contact cads@k-state.edu. 


\section{Author Information}

F. Yang, A. M. Parkhurst, S. Zhang, C. N. Lee, T. M. Brown-Brandl, K. G. Gebremedhin, and P. E. Hillman 


\title{
A Comparison of Analytic and Bayesian Approaches for Characterizing Thermal Hysteresis in Cattle Using Algebraic and Geometric Distances
}

\author{
F. Yang ${ }^{1}$, A. M. Parkhurst ${ }^{1}$, S. Zhang ${ }^{1}$, C. N. Lee ${ }^{2}$, T.M.Brown-Brandl ${ }^{3}$, K.G. Gebremedhin ${ }^{4}$, \\ P.E. Hillman (retired) ${ }^{4}$ \\ ${ }^{1}$ Department of Statistics, University of Nebraska - Lincoln \\ ${ }^{2}$ Department of Human Nutrition, Food and Animal Sciences, University of Hawaii-Manoa \\ ${ }^{3}$ USDA-ARS U.S. Meat Animal Research Center \\ ${ }^{4}$ Department of Biological and Environmental engineering, Cornell University
}

\begin{abstract}
A high ambient temperature poses a serious threat to cattle. Above a certain threshold, an animal's body temperature ( $\mathrm{Tb}$ ) appears to be driven by the hot cyclic air temperature (Ta) and hysteresis occurs. Elliptical hysteresis describes the output of a process in response to a simple harmonic input, and the trajectory forms a closed loop. The hysteresis loop shows a rotated elliptical pattern which depends on the lag between $\mathrm{Tb}$ and Ta. The objectives of this study are 1) to characterize hysteresis using bootstrapped ellipse specific nonlinear least squares 2) to reformulate models using the Bayesian method, and 3) to assess the contribution of the Bayesian approach by comparing the risks using two metrics: algebraic and geometric. Comparisons and illustrations are made using simulations over three levels of signal strength. For each method; bootstrap and Bayes, both algebraic and geometric distances are compared based on the root mean square distance (RMSE) from fitting the hysteresis loop. Data from a heat stressed steer in a field experiment was analyzed to illustrate and compare the results from each method.
\end{abstract}

\section{Introduction}

The input-output trajectory of a hysteresis system displays the dependence of the current value on past history. Elliptical hysteresis describes the behavior of a hysteretic process in response to periodic variations of a single input. In animal science, hysteresis is important when animals are thermally challenged since body temperature $(\mathrm{Tb})$ shows a dramatic response to heat stress, (HS). Studies show $\mathrm{Tb}$ is essentially unresponsive to air temperature (Ta) until a threshold is reached and then there is a dramatic response to increasing air temperature; and hysteresis becomes prominent. (Hahn, Parkhurst, \& Gaughan, 1997; Mader, et al., 2001)During heat stress, the dynamics can be characterized by thermal hysteresis which follows an elliptical loop. It measures and depicts the amount of heat stress an animal experiences in a hot environment.(Parkhurst, Model for Understanding Thermal Hysteresis During Heat Stress: A Matter of Direction, 2010) 
A phase diagram for $\mathrm{Tb}$ versus Ta indicates the time-dependence and memory associated with the hysteretic behavior; thus, plotting a phase diagram between $\mathrm{Tb}$ and $\mathrm{Ta}$ provides a way to illustrate the existence of thermal hysteresis. Thermal hysteresis becomes prominent once a delay (or lag) between $\mathrm{Tb}$ and $\mathrm{Ta}$ is noticeable in the $\mathrm{Tb}$-Ta diagram and the supposition that $\mathrm{Ta}$ is the driving force when an animal is thermally challenged appears plausible. (Figure 1)

Figure 1. Left: A time series plot shows a 4 hour lag between $\mathrm{Tb}$ and $\mathrm{Ta}$ for an animal in the temperature controlled chamber. Right: The diagram of $\mathrm{Tb} \sim \mathrm{Ta}$ for this animal shows a closed loop representing thermal hysteresis and the characteristics of the Elliptical Hysteresis Loop
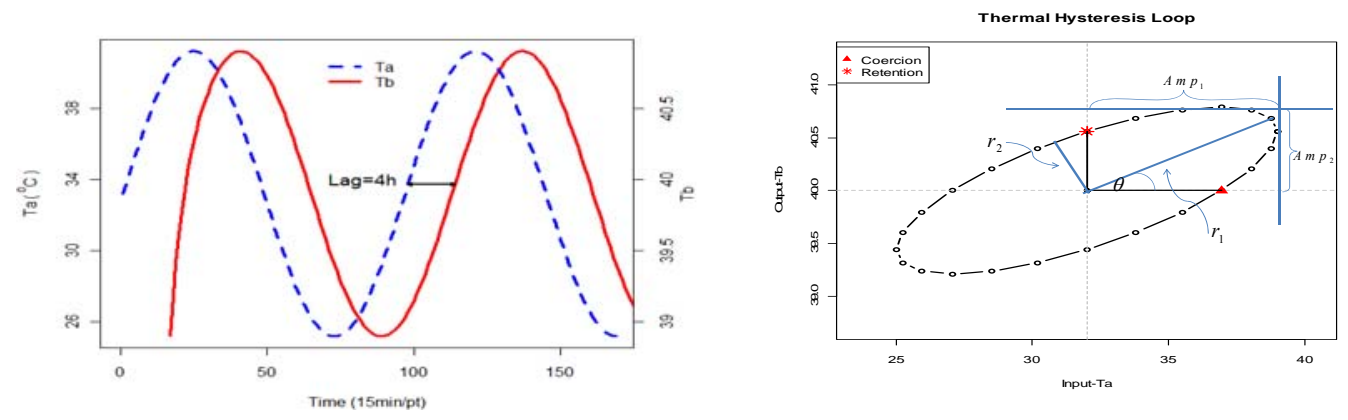

Since the thermal hysteresis loop shows a rotated elliptical pattern when input is sinusoidal, modeling the rotated ellipse is important to estimating the characteristics of the hysteresis system. An ellipse can be represented using a pair of first-order differential equations:

$$
\frac{d y}{d t}=\frac{A m p_{b}}{A m p_{a}} x \quad \frac{d x}{d t}=-\frac{A m p_{a}}{A m p_{b}} y
$$

After rotating the ellipse counter-clockwise over an angle $\theta$ and translating to the centroid $\left(\mathrm{c}_{\mathrm{a}}, \mathrm{c}_{\mathrm{b}}\right)$, the ellipse can be reparameterized as

$$
\alpha(t)=\left(A m p_{a} \cos (t) \quad A m p_{b} \sin (t)\right)
$$

By solving the differential equations, an ellipse centered at the origin can be obtained parametrically as

$$
\left(\begin{array}{l}
x(t) \\
y(t)
\end{array}\right)=\left(\begin{array}{l}
\lambda_{1} \cos (\theta) \cos (t)-\lambda_{2} \sin (\theta) \sin (t)+c_{a} \\
\lambda_{1} \sin (\theta) \cos (t)+\lambda_{2} \cos (\theta) \sin (t)+c_{b}
\end{array}\right)
$$

Where $\lambda_{1}$ and $\lambda_{2}$ are the major and minor axes of the ellipse after rotation so that

$$
A m p_{a}^{2}=\left(\lambda_{1} \cos (\theta)\right)^{2}+\left(\lambda_{2} \sin (\theta)\right)^{2} \text { and } A m p_{b}^{2}=\left(\lambda_{1} \sin (\theta)\right)^{2}+\left(\lambda_{2} \cos (\theta)\right)^{2}
$$

Several features of the hysteretic elliptical loop characterize the dynamics of heat stress. The amplitudes of $\mathrm{Ta}$ and $\mathrm{Tb}$ indicate the highest or lowest temperature that animals would experience. The center of the loop represents the averages $\left(\mathrm{c}_{\mathrm{a}}, \mathrm{c}_{\mathrm{b}}\right)$. Retention, the departure of $\mathrm{Tb}$ from the centroid indicates the level of heat left in the body as the thermal challenge recedes. It is the buildup of heat at the average input challenge. Coercion, the divergence of Ta from the centroid, indicates the strain Ta places on the animal's thermoregulation process. Alternatively, 
coercion can be thought of as a measure of the resistance of the body to heat up by increasing Ta. The angle of rotation in a hysteresis ellipse is the angle between ellipse's major axis and the Ta (horizontal)-axis. It indicates the length of the time delay; i.e. how long Tb lags Ta. The lag can be estimated from the angle of rotation of an elliptical loop. The area inside the hysteresis loop equals the work done in one period or cycle (Brokate \& Sprekels, 1996). Thus, the area of the ellipse formed during thermal hysteresis is an indication of the animal's heat load. As a result, it is possible to fit an ellipse for not only predicting $\mathrm{Tb}$ by identifying the lag of $\mathrm{Tb}$ on the current Ta, but also for estimating the amount of head load.

In this study, the ellipse is fit by incorporating both bootstrap and Bayes methods to the ellipsespecific nonlinear model. The root mean squared errors are quantified by two metrics: algebraic and geometric distance. Therefore, the objectives of this study are 1) to characterize hysteresis using bootstrapped ellipse specific nonlinear least squares 2) to reformulate the model using the Bayesian analysis, and 3) to assess the contribution of the Bayesian approach by comparing the risks using two metrics: algebraic and geometric.

\section{Methods}

\subsection{Ellipse Specific Nonlinear Least Square Method(ES-NLS)}

Developed from (1.3), a rotated ellipse with rotation angle $\theta$ and centered at $\left(c_{a}, c_{b}\right)$ was written parametrically by input $(\mathrm{x})$ and output (y). Therefore, a nonlinear statistical model of 5 fundamental parameters can be used to describe the relationship between the input and output series.

$$
\xi=\frac{2\left(\cos (\theta)\left(x-c_{a}\right)+\sin (\theta)\left(y-c_{b}\right)\right)^{2}}{A m p_{a}^{2}+A m p_{b}^{2}+\frac{A m p_{a}^{2}-A m p_{b}^{2}}{\cos ^{2} \theta-\sin ^{2} \theta}}+\frac{2\left(-\sin (\theta)\left(x-c_{a}\right)+\cos (\theta)\left(y-c_{b}\right)\right)^{2}}{A m p_{a}^{2}+A m p_{b}^{2}-\frac{A m p_{a}^{2}-A m p_{b}^{2}}{\cos ^{2} \theta-\sin ^{2} \theta}}-1+\varepsilon
$$

Equation 2.1 is called the parameterized ellipse model. The expected surface of the model is $E(\xi)=0$.Therefore, the 5 fundamental parameters can be found directly from the nonlinear model. The other 4 parameters we need to characterize the dynamics can be derived as (2.2). 


$$
\begin{aligned}
& A_{H L}=\text { Area of ellipse }=\pi * \sqrt{A m p_{a}^{2}+A m p_{b}^{2}+\frac{A m p_{a}^{2}-A m p_{b}^{2}}{\cos ^{2} \theta-\sin ^{2} \theta}} * \sqrt{A m p_{a}^{2}+A m p_{b}^{2}-\frac{A m p_{a}^{2}-A m p_{b}^{2}}{\cos ^{2} \theta-\sin ^{2} \theta}}
\end{aligned}
$$

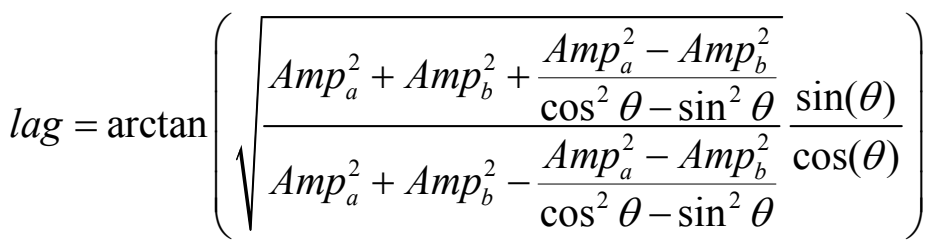

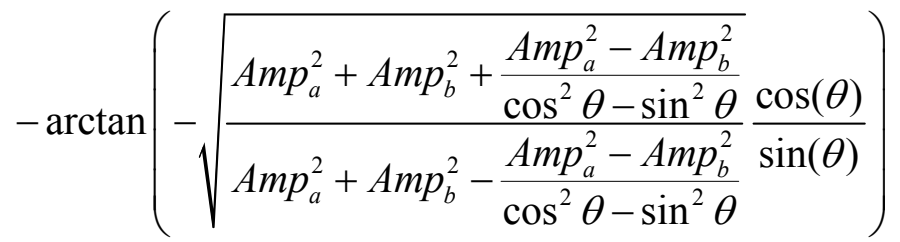

$$
\begin{aligned}
& \mathrm{R}=\sqrt{\left(A m p_{a}^{2}+A m p_{b}^{2}+\frac{A m p_{a}^{2}-A m p_{b}^{2}}{\cos ^{2} \theta-\sin ^{2} \theta}\right) \cos ^{2}(\theta)+\left(A m p_{a}^{2}+A m p_{b}^{2}-\frac{A m p_{a}^{2}-A m p_{b}^{2}}{\cos ^{2} \theta-\sin ^{2} \theta}\right) \sin ^{2}(\theta)} \\
& \mathrm{C}=\sqrt{\left(A m p_{a}^{2}+A m p_{b}^{2}+\frac{A m p_{a}^{2}-A m p_{b}^{2}}{\cos ^{2} \theta-\sin ^{2} \theta}\right) \sin ^{2}(\theta)+\left(A m p_{a}^{2}+A m p_{b}^{2}-\frac{A m p_{a}^{2}-A m p_{b}^{2}}{\cos ^{2} \theta-\sin ^{2} \theta}\right) \cos ^{2}(\theta)}
\end{aligned}
$$

\subsubsection{Starting Values and Self-starter Function}

A self-starter function described by Yang and Parkhurst (2011) was used based for the ellipsespecific direct least squares method. For the ellipse-specific method, the algebraic distance is minimized over the set of $\mathrm{n}$ points in the least squares sense that is

$$
\hat{d}^{2}=\min _{a}\left\{\sum_{i=1}^{N}\left(\left(\begin{array}{lll}
x_{i} & y_{i} & 1
\end{array}\right)\left(\begin{array}{ccc}
a_{1} & a_{2} / 2 & a_{4} / 2 \\
& a_{3} & a_{5} / 2 \\
& & a_{6}
\end{array}\right)\left(\begin{array}{c}
x_{i} \\
y_{i} \\
1
\end{array}\right)\right)\right\}
$$

with the constraint $a_{2}^{2}-4 a_{1} a_{3}<0$.

Hence, a generalized eigen-system (2.3) can help to determine an unique ellipse fitted to the data.

$$
a^{t} D^{\prime} D a-\lambda a^{t} C a=0
$$

where $D=\left(\begin{array}{cccccc}x_{1}^{2} & x_{1} y_{1} & y_{1}^{2} & x_{1} & y_{1} & 1 \\ \ldots & \ldots & \ldots & \ldots & \ldots & \ldots \\ x_{n}^{2} & x_{n} y_{n} & y_{n}^{2} & x_{n} & y_{n} & 1\end{array}\right), \quad C=\left(\begin{array}{cccccc}0 & 0 & -2 & 0 & 0 & 0 \\ 0 & 1 & 0 & 0 & 0 & 0 \\ -2 & 0 & 0 & 0 & 0 & 0 \\ 0 & 0 & 0 & 0 & 0 & 0 \\ 0 & 0 & 0 & 0 & 0 & 0 \\ 0 & 0 & 0 & 0 & 0 & 0\end{array}\right), \tilde{a}=\left(\begin{array}{c}a_{1} \\ \vdots \\ a_{6}\end{array}\right)$. 
$\tilde{a}$, which is the eigenvector corresponding to the negative eigenvalue in the eigen-system, is the solution to $(2.3)$

$$
\tilde{a}=\operatorname{Kernel}\left\{\lambda<0: \operatorname{det}\left|D^{\prime} D-\lambda C\right|\right\}
$$

The starting values for 5 fundamental parameters can be obtained from $\tilde{a}$.

$$
\begin{aligned}
& c_{a_{-} \text {start }}=\frac{2 a_{3} a_{4}-a_{2} a_{5}}{a_{2}^{2}-4 a_{1} a_{3}} \quad A m p_{a_{-} \text {start }}=\sqrt{\frac{a_{1} c_{a}^{2}+a_{2} c_{a} c_{b}+a_{3} c_{b}^{2}-a_{6}}{a_{1} \cos ^{2} \theta+a_{2} \sin \theta \cos \theta+a_{3} \sin ^{2} \theta}} \\
& c_{b_{-} \text {start }}=\frac{2 a_{1} a_{5}-a_{2} a_{4}}{a_{2}^{2}-4 a_{1} a_{3}} \quad A m p_{b_{-} \text {start }}=\sqrt{\frac{a_{1} c_{a}^{2}+a_{2} c_{a} c_{T b}+a_{3} c_{b}^{2}-a_{6}}{a_{1} \sin ^{2} \theta-a_{2} \sin \theta \cos \theta+a_{3} \cos ^{2} \theta}} \quad \theta_{\text {start }}=\frac{1}{2}\left(\arctan \frac{a_{2}}{a_{1}-a_{3}}\right)
\end{aligned}
$$

\subsection{Ellipse Specific Nonlinear Least Square with Bootstrap Method (ES-NLS-Boot)}

Bootstrapping developed by Efron $(1979 ; 1993)$ provides an alternative way of calculating SEs. It allows us to estimate the population distribution of the parameter estimates. (R package: Boot) By resampling the residuals from ellipse specific nonlinear least square method, subset of each simulated realization was drawn randomly with replacement. For each of the 1000 simulated realizations, 999 bootstrapping iterations were performed on the ellipse-specific nonlinear least squares method. Bootstrapping estimates and SEs for each parameter were obtained by averaging over the total number of iterations. (Figure 2)

Figure 2. Diagram of Bootstrapping procedure

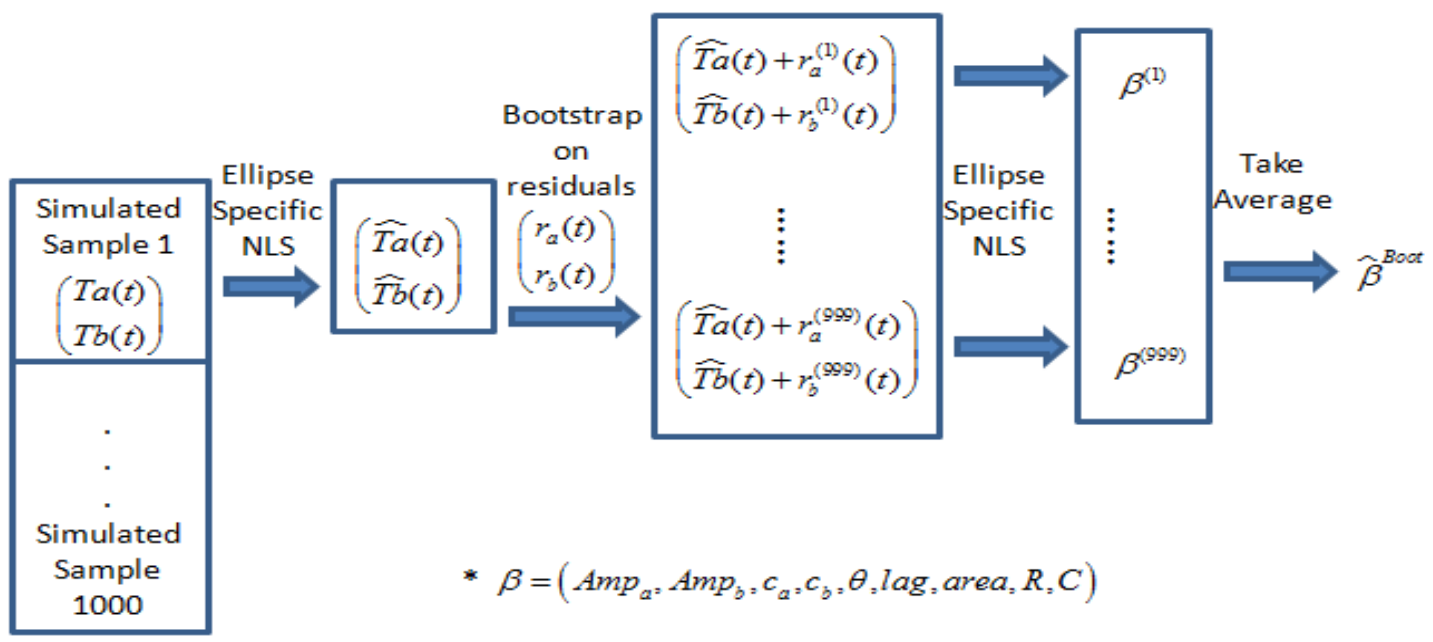




\subsection{Ellipse Specific Nonlinear Least Square with Bayes Method(ES-NLS-Bayes)}

For both the ES-NLS and ES-NLS-Boot methods, all the parameters were viewed as fixed. However, in some situations such as uncontrolled air temperature, some parameters may be viewed as random quantities. In such case, Markov chain Monte Carlo method (MCMC) (Carlin, 2009) provides a good way to fit data. In this study, one of the MCMC methods, Gibbs sampler with Metropolis, is applied to ES-NLS when assume amplitude of $\mathrm{Tb}\left(\mathrm{Amp}_{\mathrm{b}}\right)$ is randomly distributed.

In ES-NLS_Bayes, we assume the ellipse follows a distribution as

$$
z=f(x, y)=\xi_{i}+\varepsilon
$$

where $\xi_{i}=\frac{2\left(\cos (\theta)\left(x_{i}-c_{a}\right)+\sin (\theta)\left(y_{i}-c_{b}\right)\right)^{2}}{A m p_{a}^{2}+A m p_{b}^{2}+\frac{A m p_{a}^{2}-A m p_{b}^{2}}{\cos ^{2} \theta-\sin ^{2} \theta}}+\frac{2\left(-\sin (\theta)\left(x_{i}-c_{a}\right)+\cos (\theta)\left(y_{i}-c_{b}\right)\right)^{2}}{A m p_{a}^{2}+A m p_{b}^{2}-\frac{A m p_{a}^{2}-A m p_{b}^{2}}{\cos ^{2} \theta-\sin ^{2} \theta}}-1$ and $\varepsilon$ is the random error for the whole model.

The conditional distribution of $z$ given Amp $\mathrm{p}_{\mathrm{b}}$ is derived as

$$
z_{i} \mid A m p_{b} \sim N\left(\xi_{i}, \sigma^{2}\right)
$$

with prior

$$
A m p_{b} \sim \operatorname{Gamma}\left(a_{2}, b_{2}\right)
$$

Hence, the posterior distribution of $\mathrm{Amp}_{\mathrm{b}}$ is

$$
p\left(A m p_{b} \mid z\right) \propto h\left(A m p_{b}\right) \propto A m p_{b}{ }^{a_{2}} \exp \left(\frac{\sum \xi_{i}^{2}}{-2 \sigma^{2}}-\frac{A m p_{b}}{b_{2}}\right)
$$

The posterior distribution of $A m p_{b}$ can be obtained using the Gibbs sampler with Metropolis, which is shown as follows.

Gibbs sampler with Metropolis

Step 0: Give starting values for Amp

Step 1 to T: Draw $A m p_{b}^{*}$ from a proposal density q(.). In this study, the proposal density $q()=.N\left(A m p_{b}^{(t-1)}, \sigma^{2}\right)$. Then, $A m p_{b}^{*} \sim q\left(A m p_{b}^{(t-1)}\right)$

Compute

$$
r=\frac{h\left(A m p_{b}^{*}\right) / q\left(A m p_{b}^{*}\right)}{h\left(A m p_{b}^{(t-1)}\right) / q\left(A m p_{b}^{(t-1)}\right)}
$$


If $r \geq 1$, set $A m p_{b}^{(t)}=A m p_{b}^{*}$.

If $r<1$, set $A m p_{b}^{(t)}=\left\{\begin{array}{c}A m p_{b}^{*}, \text { with prob } r \\ A m p_{b}^{(t-1)}, \text { with prob } 1-r\end{array}\right.$

Repeat the above steps for $m$ chains. After $\mathrm{a}_{0}$ burn-in period, the posterior mean for $\mathrm{Amp}_{\mathrm{b}}$ is

$$
\hat{E}\left(A m p_{b} \mid \mathbf{z}\right)=\frac{1}{m\left(T-t_{0}\right)} \sum_{j=1}^{m} \sum_{t=t_{0}+1}^{T}\left(A m p_{b}\right)_{j}^{(t)}
$$

In this study, $\mathrm{m}=5$ (chains), $\mathrm{T}=10000$ (total iterations), $\mathrm{t}_{0}=2000$ (burn-in).

\subsection{Comparisons}

The ES-NLS-Boot and ES-NLS-Bayes methods were compared by root mean squared of residuals (RMSE). In this study, RMSE and SEs for every parameter were obtained by two metrics: algebraic distance and geometric distance. Comparisons were made between two metrics and also between methods. The method with smaller RMSE and SEs would be preferred.

\subsubsection{Algebraic distance}

The algebraic distance is a linear distance metric. In $\mathfrak{R}^{2}$, if a closed curve can be defined implicitly as $f(x, y)=0$, where $(\mathrm{x}, \mathrm{y})$ is the point on the curve, then the algebraic distance from a point $\left(x_{k}, y_{k}\right)$ to the closed curve is

$$
D_{\mathrm{A}}=f\left(x_{k}, y_{k}\right)
$$

Since a rotated ellipse with rotation angle $\theta$ and centered at $\left(\mathrm{c}_{\mathrm{x}}, \mathrm{c}_{\mathrm{y}}\right)$ was written parametrically as (2.1), the algebraic distance from a point $\left(x_{k}, y_{k}\right)$ to this ellipse is

$$
D_{\mathrm{A}}\left(x_{k}, y_{k}\right)=\frac{2\left(\cos (\theta)\left(x_{k}-c_{T a}\right)+\sin (\theta)\left(y_{k}-c_{T b}\right)\right)^{2}}{A m p_{T a}^{2}+A m p_{T b}^{2}+\frac{A m p_{T a}^{2}-A m p_{T b}^{2}}{\cos ^{2} \theta-\sin ^{2} \theta}}+\frac{2\left(-\sin (\theta)\left(x_{k}-c_{T a}\right)+\cos (\theta)\left(y_{k}-c_{T b}\right)\right)^{2}}{A m p_{T a}^{2}+A m p_{T b}^{2}-\frac{A m p_{T a}^{2}-A m p_{T b}^{2}}{\cos ^{2} \theta-\sin ^{2} \theta}}-1
$$

Under the algebraic distance metric, the root mean squared residual is

$$
R M S E_{\mathrm{A}}=\sum_{i=1}^{n} D_{\mathrm{A}}^{2}\left(x_{i}, y_{i}\right)
$$




\subsubsection{Geometric distance}

Geometric distance indicates the shortest distance from a point to a plane in $\mathfrak{R}^{n}$. It is also called Euclidean distance. In a univariate study, the geometric distance, which represents the departure (residual) from observed points to predicted points, is measured using vertical distance. i.e. $D_{G}=y_{o b s}-\hat{y}$ (Figure 3, left). However, in a multivariate study, vertical distance is not adequate. Information of other dimensions is lost if only vertical distance is used. (Figure 3 middle left). In order to gain more information, geometric distance is important for estimating the variation from one point to a curve or a surface.

Suppose $\mathbf{Y}^{\mathrm{n}}$ is a point in $\mathfrak{R}^{n}$, then the geometric distance is the vertical distance from $\mathbf{Y}^{\mathrm{n}}$ to the expectation surface. i.e. $Y^{n}-\hat{Y}^{n}$. (Figure 3 middle right) In this study, geometric distance is the distance between the observed point and its projection on the tangent plane on the closed curve(Figure 3 right),

$$
D_{G}=\min _{(\hat{x}, \hat{y})}\{\|(x, y)-(\hat{x}, \hat{y})\|: f(\hat{x}, \hat{y})=0\}
$$

Figure 3 Illustration of geometric distances. From left to right: univariate case; vertical distance in bivariate case; geometric distance in general case (expectation surface); geometric distance in bivariate case.
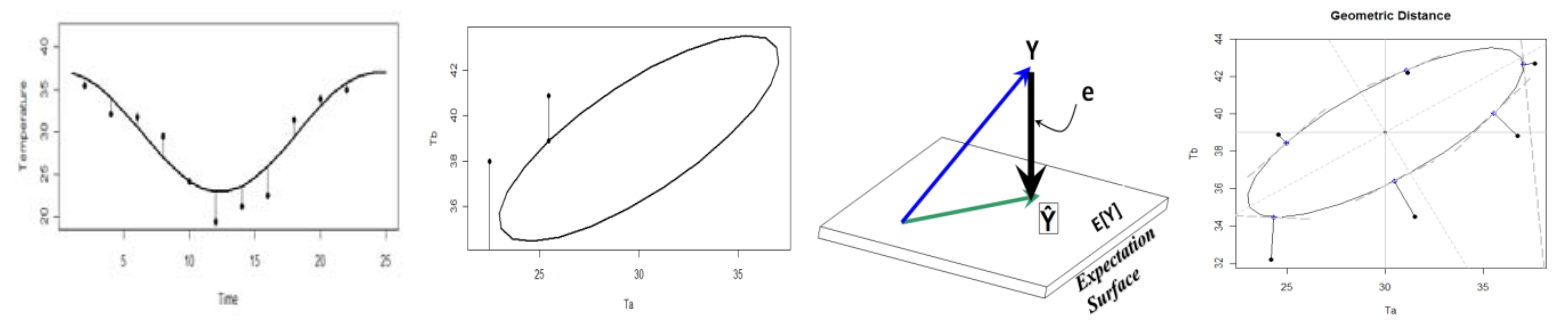

Using the geometric distance, the root mean squared residual is

$$
R M S E_{\mathrm{G}}=\sum_{i=1}^{n} D_{G}^{2}\left(x_{i}, y_{i}\right)
$$

\subsection{2.i Gauss Newton's method for obtaining Geometric Distance}

Suppose $\left(x_{k}, y_{k}\right)$ is an observation at point $\mathrm{t}$, and $\mathrm{d}$ is the geometric distance from $\left(x_{k}, y_{k}\right)$ to an ellipse, then

$$
d^{2}=\left(x(t)-x_{k}\right)^{2}+\left(y(t)-y_{k}\right)^{2}
$$


Let $\frac{\partial\left(d^{2}\right)}{\partial t}=0$, then a function $\mathrm{f}$ about $\mathrm{t}$ forthe input and output series can be obtained

$$
f(t)=2\left(x(t)-x^{*}\right) x^{\prime}(t)+2\left(y(t)-y^{*}\right) y^{\prime}(t)
$$

The Jacobian matrix, $\mathrm{J}$, is

$$
J=\left(\frac{\partial f}{\partial \vec{x}}\right)=\left(\begin{array}{ll}
\frac{\partial f}{\partial x} & \frac{\partial f}{\partial y}
\end{array}\right)
$$

The geometric distance can be found using Gauss Newton's method. (Ahn, Rauh, \& Warnecke, 2001)

Gauss Newton's method is an iterative method. The procedure for finding the corresponding tangent point $\left(x^{*}, y^{*}\right)$ starts with two points $\left(x_{k 1}, y_{k 1}\right)$ and $\left(x_{k 2}, y_{k 2}\right)$ so that $\left(x_{k 1}, y_{k 1}\right)$ is the intersect of the ellipse and the line through the observation and centroid and $\left(x_{k 2}, y_{k 2}\right)$ is the intersect of the ellipse and the line through the observation but orthogonal to the major axis. (Figure 4)

Then, the middle point $\left(x_{k 0}, y_{k 0}\right)$ between $\left(x_{k 1}, y_{k 1}\right)$ and $\left(x_{k 2}, y_{k 2}\right)$ is used as the initial point for finding the tangent point,

$$
\left(x_{k 0}, y_{k 0}\right)=\left(\frac{x_{k 1}+x_{k 2}}{2}, \frac{y_{k 1}+y_{k 2}}{2}\right)
$$

For step $i=1,2,3, \ldots$

$$
\begin{aligned}
& (\Delta x, \Delta y)=J^{-1} f\left(x_{k 0}, y_{k 0}\right) \\
& \left(x_{i}, y_{i}\right)=\left(x_{i-1}, y_{i-1}\right)+(\Delta x, \Delta y)
\end{aligned}
$$

The iteration converges at some step $\mathrm{n}$ if

$$
\left(\left|\frac{x_{n+1}-x_{n}}{x_{n}}\right|,\left|\frac{y_{n+1}-y_{n}}{y_{n}}\right|\right)<\varepsilon
$$

with a specified convergence tolerance $\varepsilon=(1 \mathrm{e}-6,1 \mathrm{e}-6)$.

Therefore, the tangent point

$$
\left(x^{*}, y^{*}\right)=\left(x_{n}, y_{n}\right)
$$


Figure 4. Illustration of Gauss Newton's method for finding geometric distance

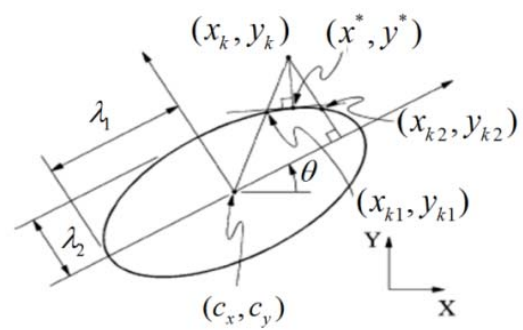

\section{Data}

\subsection{Simulation}

Three simulation studies are conducted to evaluate the performance of NLS-Boot and NLSBayes. Realistic numbers for the model parameters based on values reported in previous literature(Hillman, Gebremedhin, Brown-Brandl, \& Lee, 2005)were used. Assume an input heat challenge of $24 \pm 8{ }^{\circ} \mathrm{C}$ is applied resulting in an output signal of $39 \pm 0.6^{\circ} \mathrm{C}$ with a $5.1 \mathrm{hr}$ lag. The true area of the ellipse is 14.67 , the true retention (R) is $0.58{ }^{\circ} \mathrm{C}$, and the true coercion (C) is 7.78 ${ }^{\circ} \mathrm{C}$. The 1000 datasets were simulated by adding errors to both $\mathrm{Ta}\left(\varepsilon_{a}\right)$ and $\mathrm{Tb}\left(\varepsilon_{b}\right)$ such that three signal-to-noise ratios (SNR) levels (weak, moderate, strong) were generated. In strong SNR, $\varepsilon_{\mathrm{b}}$ is 0.03 and the corresponding relative variation $\left(R V=A m p_{a} / \varepsilon_{b}\right.$, where $\left.A m p_{a}=8\right)$ is 267 ; in moderate $\mathrm{SNR}, \varepsilon_{\mathrm{b}}$ is 0.05 and the corresponding RV is 160 ; in weak $\mathrm{SNR}, \varepsilon_{\mathrm{b}}$ is 0.13 and the corresponding RV is 62. For each study, the covariance matrices based on algebraic and geometric distance were constructed. Hence, six covariance matrices were built in the simulation study to compare the NLS-Boot and NLS-Bayes methods.

\subsection{Field Experiment}

A field experimental was conducted over two weeks (from end of July to beginning of August) in 2004 at USDA-ARS U.S. Meat Animal Research Center Nebraska. Thirty-two heifers were randomly selected in order to study the effect of accessibility to shade among four breeds of steers. The $\mathrm{Ta}$ and $\mathrm{Tb}$ were recorded hourly. In this article, an Angus steer with black hair coat and without access to shade for one hot experimental day was selected, and its $\mathrm{Tb}$ and corresponding Ta were used to illustrate the NLS-Boot and NLS-Bayes methods.

\section{Results}

\subsection{Simulation results}


Overall, the geometric distance always produces the smallest RMSE; as the data becomes nosier (i.e. SNR becomes weaker and RV decreases), both the algebraic and geometric RMSEs increase (Table 1). The Bayes method provides smaller RMSEs than the bootstrap method for both metrics. Both methods underestimate the true algebraic RMSE whereas, both methods overestimate the true geometric RMSEs for all three SNR levels.

Table 1. RMSE by Algebraic distance and Geometric distance for Bayes and Bootstrap methods under Strong (S), Median (M), and Low (L) SNR levels.

\begin{tabular}{|l|l|l|l|l|}
\hline Estimate & SNR & True & Bayes & Bootstrap \\
\hline $\begin{array}{l}\text { RMSE } \\
\text { (Algebraic) }\end{array}$ & $\mathrm{S}$ & .117 & .088 & .101 \\
\hline & $\mathrm{M}$ & .172 & .138 & .139 \\
\hline & $\mathrm{W}$ & .364 & .208 & .241 \\
\hline $\begin{array}{l}\text { RMSE } \\
\text { (Geometric) }\end{array}$ & $\mathrm{S}$ & .038 & .051 & .057 \\
\hline & $\mathrm{M}$ & .057 & .076 & .089 \\
\hline & $\mathrm{W}$ & .112 & .133 & .150 \\
\hline
\end{tabular}

Table 2 shows that while the methods provide similar estimates, the estimates provided by NLSBayes are slightly closer to the truth than NLS-Boot. Morover, the NLS-Boot estimates become more biased as noise increases. The estimates for Amp under three SNRs are all over-estimated for both methods. The estimates become more biased as the noise increases which causes the estimates for the derived parameters: area, lag and retention to be overestimated, while the estimates for coercion are underestimated.

The SE's for each parameter increase with noise (SNR level) and the geometric distance always produces the smallest SE no matter which method is used. When considering which method gives SE's closest to the truth, we find the Bayes method gives geometric SE's closer to the truth while the bootstrap method gives SE's closer to the truth for the algebraic metric. This is because the Bayes method produces the RMSEs which are closer to the truth for the geometric metric and the Bootstrap method produces the RMSEs which is closer to the truth for the algebraic metric. Both methods underestimate the SE's. 
Table 2. Estimates of Amp , and Derived Parameters: Area, Lag, Retention (R), and Coercion (C) and their SEs by Algebraic and Geometric distances using both Bayes and Bootstrapping methods under strong (S), median (M), and low (L) SNR levels.

\begin{tabular}{|c|c|c|c|c|c|c|c|c|c|c|}
\hline \multirow{2}{*}{$\begin{array}{l}\text { Param } \\
\text { eter }\end{array}$} & \multirow{2}{*}{ 艺 } & \multicolumn{3}{|c|}{ Estimate } & \multicolumn{3}{|c|}{ SE (Algebraic) } & \multicolumn{3}{|c|}{ SE (Geometric) } \\
\hline & & True & Bayes & Bootstrap & True & Bayes & Bootstrap & True & Bayes & Bootstrap \\
\hline \multirow[t]{3}{*}{$A m p_{b}$} & $\mathrm{~S}$ & \multirow{3}{*}{.6} & .624 & .628 & .015 & .011 & .013 & .004 & .006 & .007 \\
\hline & M & & $.646 \#$ & $.651 \#$ & .022 & .018 & .018 & .009 & .012 & .014 \\
\hline & W & & .729 & .738 & .058 & .033 & .038 & .018 & .021 & .024 \\
\hline \multirow[t]{3}{*}{ Area } & $\mathrm{S}$ & \multirow{3}{*}{14.67} & 15.08 & 15.10 & .406 & .305 & .350 & .132 & .177 & .198 \\
\hline & $\mathrm{M}$ & & 15.54 & 15.56 & .624 & .501 & .505 & .243 & .324 & .379 \\
\hline & W & & 17.21 & 17.24 & 1.50 & .854 & .989 & .461 & .547 & .617 \\
\hline \multirow[t]{3}{*}{ Lag } & $\mathrm{S}$ & \multirow{3}{*}{5.1} & 5.16 & 5.19 & .146 & .110 & .126 & .048 & .064 & .072 \\
\hline & $\mathrm{M}$ & & 5.23 & 5.36 & .223 & .179 & .180 & .087 & .116 & .136 \\
\hline & W & & 5.38 & 5.67 & .492 & .281 & .326 & .152 & .180 & .203 \\
\hline \multirow[t]{3}{*}{$\mathrm{R}$} & $\mathrm{S}$ & \multirow{3}{*}{.58} & .61 & .62 & .015 & .011 & .013 & .004 & .006 & .007 \\
\hline & M & & $.63 \#$ & $.66 \#$ & .022 & .018 & .018 & .009 & .012 & .014 \\
\hline & W & & $.71 \#$ & $.79 \#$ & .054 & .031 & .036 & .017 & .020 & .023 \\
\hline \multirow[t]{3}{*}{$\mathrm{C}$} & $\mathrm{S}$ & \multirow{3}{*}{7.78} & 7.71 & 7.69 & .176 & .132 & .152 & .057 & .077 & .086 \\
\hline & M & & 7.66 & 7.62 & .256 & .205 & .206 & .099 & .132 & .155 \\
\hline & $\mathrm{W}$ & & 7.51 & 7.49 & .504 & .288 & .334 & .156 & .185 & .209 \\
\hline
\end{tabular}

\subsection{Field experiment results}

Table 3 gives the results for a heat stress day for one Angus steer without access to shade. The SNR level for this data is moderated. As expected from the simulation study, the geometric metric distance produces smaller RMSE and SE's. The NLS-Boot and NLS-Bayes returned similar estimates with the notable exception of heat load (area). The simulation study indicates geometric SEs provided by NLS-Bayes are slightly closer to the truth, NLS-Boot results are used for the ease of implementation issue. Therefore, the selected black Angus steer reached a maximum challenge in $\mathrm{Tb}(0.7 \pm .03 \mathrm{C})$ after being exposed to a maximum increment in $\mathrm{Ta}$

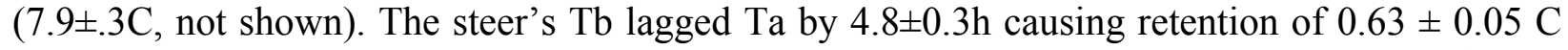
and coercion of $7.50 \pm 0.34 \mathrm{C}$. The heat load given by area is estimated to be $15.66 \pm 0.77 \mathrm{C}^{2}$. 
Table 3. Estimates of Amp , Area, Lag, Retention (R), and Coercion (C), their SEs and RMSE by Algebraic (A) and Geometric $(\mathrm{G})$ distances using both Bayes and Bootstrapping methods for a Heat Stressed Black Angus Steer.

\begin{tabular}{|l|l|l|l|l|l|l|}
\hline \multirow{2}{*}{ Parameter } & \multicolumn{3}{|l}{ Bayes } & \multicolumn{3}{l}{ Bootstrap } \\
\cline { 2 - 7 } & Est & SE(A) & SE(G) & Est & SE(A) & SE(G) \\
\hline Ampb & .7 & .04 & .03 & .7 & .05 & .04 \\
\hline Area & 15.8 & .89 & .72 & 15.7 & .95 & .77 \\
\hline Lag & 4.8 & .31 & .25 & 4.8 & .39 & .32 \\
\hline R & .6 & .03 & .03 & .6 & .06 & .05 \\
\hline C & 7.5 & .26 & .21 & 7.5 & .42 & .34 \\
\hline RMSE(A) & .213 & & & .214 & & \\
\hline RMSE(G) & .172 & & & .174 & & \\
\hline
\end{tabular}

\section{Conclusion}

geometric distance is recommended for bivariate NLS. Geometric distance provides the smallest RMSE and SE's. It gives an alternative way to estimate hysteresis loop by minimizing geometric sum squared of residuals. Hence, Both Bayes and Bootstrap can be combined with ellipse specific nonlinear model for fitting the elliptical hysteresis loop. Both methods produce similar estimates. For the five examined parameters, only coercion is under estimated but all other parameters are over estimated. Bayes estimates are slightly closer to the truth especially as SNR increases and may be explored further. However, NLS-Boot has the advantage that it is easier to implement.

For future study, the NLS-Bayes method may be expanded. More fundamental parameters, such as $A_{m p}$ and $\theta$, could be assumed as random and then incorporated with the NLS-Bayes method. Developing the NLS-Bayes method may improve the analysis of datain the cases where the SNR level is weak or the process if from uncontrolled $\mathrm{Ta}$ obtained from field data (i.e. $\mathrm{Tb}$ is influenced by other environmental factors).

\section{Summary}

Thermal hysteresis elliptical loops can be estimated using ellipse-specific nonlinear least squares method. In the ellipse-specific nonlinear least squares method, both bootstrap and Bayes methods 
can be implemented in order to improve the fitting of an elliptical hysteresis loop.In NLS-boot, the residuals from ellipse specific nonlinear least square method were resampled with replacement and then 999 bootstrapping iterations were performed on the ellipse-specific nonlinear least squares method. In NLS-bayes, amplitude of Tb was assumed to be random when holding other constant. Markov Monte Carlo method was used. The posterior distribution of $\mathrm{Amp}_{\mathrm{b}}$ can be obtained using the Gibbs sampler with Metropolis.Five fundamental parameters, amplitudes of input and output, centroids, and regression angle of rotation, and four derived parameters lag, area, retention, and coercion can be estimated byeach method. Lag and area of an elliptical hysteresis loop are two features used to characterize the dynamics of heat stress. In simulation study, estimates, including RMSE from algebraic distance, RMSE from geometric distance, $\mathrm{Amp}_{\mathrm{b}}$, lag, area, retention, and coercion, and the corresponding SE were obtained. The simulation study showed there are slight differences among two methods in the quality of the parameter estimates.For bothmethods, RMSEs and SEs for all parameters usinggeometric distance were always smaller than those usingalgebraic distance.Although estimates fromNLSBayes areslightly closer to the truth in the simulation study, NLS-boot is still has the advantage that it is easier to implement.

\section{Reference}

Ahn, S., Rauh, W., \& Warnecke, H. (2001). Least-squares orthogonal distances fitting of circle, sphere, ellipse, hyperbola, and parabola. Pattern Recognition, 34 (12), 2283-2303.

Brokate, M., \& Sprekels, J. (1996). Hysteresis and Phase Transitions. Springer Verlag.

Efron, B. (1979). Bootstrap methods: another look at the jackknife. The annals of Statistics, 7 (1), $1-26$.

Efron, B., \& Tibshirani, R. (1993). An introduction to the bootstrap (Vol. 57). Chapman $\backslash \&$ Hall/CRC.

Fitzgibbon, A., Pilu, M., \& Fisher, R. (1999). Direct Least Squares Fitting of Ellipses. Pattern

Recognition, 1996., Proceedings of the 13th International Conference on, 1, 253-257.

Hahn, G., Parkhurst, A., \& Gaughan, J. (1997). Cattle Respiration Rate as A Function of

Ambient Temperature. Transactions of American Society of Agricultural Engineering, 40, 97 121.

Halir, R., \& Flusser, J. (1998). Numerically Stable Direct Least Squares Fitting of Ellipses. The Sixth International Conference in Central Europe on Computer Graphics and Visualization , 59108.

Hillman, P., Gebremedhin, K., Brown-Brandl, T., \& Lee, C. (2005). Thermal analysis and behavioral activity of heifers in shade or sunlight. St. Joseph, Mich.: ASAE, (pp. 151-161). 
Ikhouane, F., \& Rodellar, J. (2007). Systems with hysteresis: analysis, identification and control using the Bouc-Wen model. Wiley-Interscience.

Jones, H. E. (1937). The nature of regression function in the correlation analysis of time series. Econometrica.

Lapshin, R. (1995). Analytical Model for the Approximation of Hysteresis Loop and Its Application to the Scanning Tunneling Microscope. Review of Scientific Instruments, 66 (9), 4718-4730.

Mader, T., Holt, S., Gaughan, J., Hahn, G., Davis, M., Parkhurst, A., et al. (2001). Heat Load Management for Feedlot Cattle. Proceedings, Sixth International Livestock Environment Symposium. Louisville, Kentucky, 147-153.

O'Leary, P., \& Zsombor-Murray, P. (2004). Direct and Specific Least-Square Fitting of Hyperbole and Ellipses. Journal of Electronic Imaging, 13, 492.

Parkhurst, A. (2010). Model for Understanding Thermal Hysteresis During Heat Stress: A Matter of Direction. International Journal of Biometeorology, 54, 637.

Parkhurst, A., Spiers, D., Mader, T., \& Hahn, G. (2002). Spline models for estimating heat stress thresholds in cattle. Proceedings of the 14th Annual Kansas State University Conference on Applied Statistics in Agriculture.

Pilu, M., Fitzgibbon, A., \& Fisher, R. (1996). Ellipse-Specific Direct Least-Square Fitting. Image Processing, 1996. Proceedings., International Conference on, 3, 599-602.

Prowse, C. (1984). Some thoughts on lag and hysteresis. Area, 17-23.

Ritz, C., \& Streibig, J. (2008). Nonlinear regression with R. Springer Verlag.

Vargas, R., \& Allen, M. (2008). Environmental controls and the influence of vegetation type, fine roots and rhizomorphs on diel and seasonal variation in soil respiration. New Phytologist, 179 (2), 460-471.

Yang, F., \& Parkhurst, A. (2011). Estimating Area and Lag Associated with Thermal Hysteresis in Cattle. Proc. 22nd Annual Kansas State University Conference on Applied Statistics in Agriculture . 